\section{Huntingtine, mitose et destinée cellulaire}

Juliette Godin, Sandrine Humbert
Institut Curie, CNRS UMR 3306 , Inserm U1005, 91405 Orsay Cedex, France. sandrine.humbert@curie.fr

phase de synthèse (phase S) de l'ADN et se sépare pendant la mitose pour former les deux pôles du fuseau mitotique [9, 10]. Pour qu'une cellule se divise correctement, les chromosomes doivent être bien alignés. Pour cela, la position du fuseau mitotique est finement régulée. Ceci implique deux étapes : la formation et l'orientation du fuseau mitotique, dont la réussite est en partie rendue possible par des protéines telles que la dynéine et la dynactine qui, une fois ancrées au cortex de la cellule, génèrent des forces nécessaires à l'alignement du fuseau.

La huntingtine est associée au réseau microtubulaire par sa liaison à la dynéine et à la sous-unité p150 Glued de la dynactine $[2,4]$. Elle est aussi enrichie au centrosome et aux pôles du fuseau mitotique. Nous avons émis l'hypothèse selon laquelle la huntingtine pourrait participer au positionnement du fuseau mitotique. Nous avons d'abord utilisé une approche d'invalidation de la huntingtine par interférence ARN dans des cellules en culture. L'analyse de l'angle entre l'axe pôle-pôle et le plan du substrat dans des cellules d'origine neuronale sauvages ou déplétées en huntingtine a

Figure 1. La huntingtine et le fuseau mitotique dans des cellules en culture. A. Dans la majorité des cas l'axe pôle-pôle (jaune) et le plan du substrat (trait gras noir) sont parallèles. Des cellules invalidées pour la huntingtine présentent un défaut d'orientation du fuseau par rapport au plan du substrat. B. Des enregistrements de la division de cellules exprimant une tubuline fluorescente (en vert) permettent de suivre le positionnement et l'alignement du fuseau mitotique. Pôles du fuseau : étoiles blanches et jaunes. Barre d'échelle $10 \mu \mathrm{m}$. 


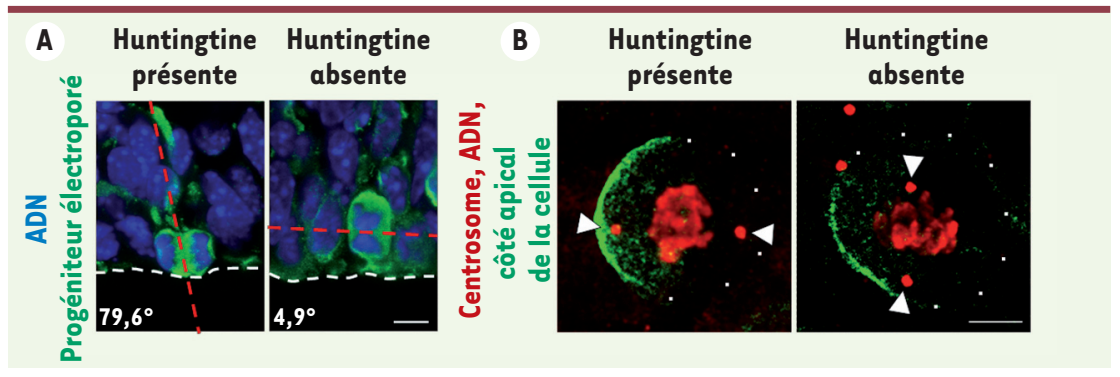

Figure 2. La huntingtine et l'orientation du fuseau : une fonction conservée chez la souris et la drosophile. A. Les cellules progénitrices du cortex murin se divisent en majorité avec un fuseau de division parallèle à la surface épithéliale (ligne pointillée blanche), ce qui correspond à un plan de clivage vertical (ligne pointillée rouge). En absence de huntingtine (après électroporation d'ARN interférent), on

observe une augmentation de la proportion de progéniteurs qui se divisent avec un plan de clivage horizontal. Barre d'échelle $10 \mu \mathrm{m}$. B. Le fuseau (flèches blanches) se positionne perpendiculairement à l'axe apical-basal (en vert) pour maintenir la polarité du neuroblaste de drosophile. La huntingtine est nécessaire au bon déroulement de cette étape. Barre d'échelle $5 \mu \mathrm{m}$.

révélé un défaut d'orientation du fuseau (augmentation de l'angle) dans ces dernières cellules (Figure 1A). La réexpression d'un fragment de huntingtine dans les cellules invalidées rétablit totalement le défaut d'orientation observé en l'absence de huntingtine.

$\varepsilon n$ l'absence de huntingtine, les fuseaux adoptent-ils lors de leur formation une position déviée ou cherchent-ils la bonne position sans la trouver? Des enregistrements de la division de cellules exprimant une tubuline fluorescente ont permis de suivre en temps réel le positionnement et I'alignement du fuseau mitotique et de mettre en évidence de plus fortes oscillations du fuseau en absence d'huntingtine (Figure 1B). Dans la plupart des cas, le résultat de ces mouvements est l'adoption par les fuseaux d'une position finale mal orientée. L'ensemble de ces résultats suggère que la huntingtine participe au bon positionnement du fuseau mitotique au cours de la division.

\section{Quels sont les mécanismes \\ moléculaires sous-jacents?}

La huntingtine stimule le transport vésiculaire le long des microtubules [2, 4]. Pour cela, elle forme un complexe avec plusieurs protéines connues pour leur fonction dans ces dynamiques intracellulaires, notamment la dynéine et la sous-unité p150 Glued de la dynactine. Ces protéines jouent également un rôle lors de la mise en place du fuseau de division et de son orientation. NuMA, une protéine mitotique nucléaire, est un autre acteur essentiel pour l'organisation des microtubules aux pôles du fuseau [6]. De fait, huntingtine, dynéine, p150 Glued et NuMA sont localisées aux pôles du fuseau de division. En absence de huntingtine, ces protéines sont dispersées : elles sont aussi nombreuses qu'en condition normale mais leur concentration au niveau du centrosome est affectée. Une telle délocalisation les empêche d'assurer leur fonction aux pôles. La huntingtine contrôlerait donc l'orientation du fuseau de division en participant à l'assemblage d'un complexe NuMA/dynéine/dynactine aux pôles de ce fuseau.

\section{La huntingtine régule}

\section{la neurogenèse in vivo}

La huntingtine est exprimée dans le cortex au cours du développement embryonnaire chez la souris. Les neurones corticaux naissent à partir de cellules souches qui se divisent de manière symétrique ou asymétrique [7]. Lorsque la division est symétrique, les cellules s'autorenouvellent (les deux cellules filles sont des cellules souches) et il y a une expansion des progéniteurs. À l'inverse, pendant la division asymétrique, il y a production d'une cellule souche et d'un neurone qui migre pour intégrer le futur cortex. L'orientation du fuseau peut, au moins en partie, influencer l'orientation de la division. Afin d'évaluer l'importance de la huntingtine dans ce processus, les auteurs ont invalidé l'expression de la huntingtine dans des cellules souches corticales d'embryons de souris et ana- lysé l'orientation du fuseau (Figure 2A). Les cellules progénitrices peuvent se diviser avec un fuseau de division parallèle, intermédiaire ou perpendiculaire à la surface épithéliale, ce qui correspond à un plan de clivage vertical $\left(60^{\circ}-90^{\circ}\right)$, intermédiaire $\left(30^{\circ}-60^{\circ}\right)$ ou horizontal $\left(0^{\circ}-30^{\circ}\right)$. L'analyse de l'angle entre le plan de clivage et la surface épithéliale a révélé une augmentation de la proportion de progéniteurs neuronaux qui se divisent avec un plan horizontal en l'absence de huntingtine par rapport à une situation contrôle.

Le plan de clivage de la division de cellules progénitrices pouvant influencer le destin cellulaire des cellules filles, le défaut d'orientation du fuseau induit par l'absence de huntingtine dans les progéniteurs pourrait moduler ce processus. Le BrdU est utilisé comme marqueur de cellules en division car il s'incorpore à I'ADN néosynthétisé. Grâce à un tel marquage, nous avons suivi le devenir de cellules filles des progéniteurs en division au moment de l'incorporation de BrdU et montré que la diminution de huntingtine conduit à une augmentation de la neurogenése. En effet, le nombre de progéniteurs générés diminue alors que le nombre de neurones différenciés augmente en absence de huntingtine. Ainsi, la perte d'expression de la huntingtine dans des progéniteurs corticaux favorise leur différenciation neuronale au détriment de leur maintien dans un statut de progéniteurs en phase de prolifération. 


\section{Une fonction conservée chez la drosophile}

Au cours de la neurogenèse chez la mouche Drosophila melanogaster, les divisions des neuroblastes, précurseurs du système nerveux central, sont asymétriques $[7,8]$. Le neuroblaste se divise pour donner deux cellules de tailles et de compositions différentes: une cellule ganglionnaire et un neuroblaste. L'alignement du fuseau le long de l'axe apical-basal en prométaphase est essentiel pour maintenir la polarité du neuroblaste (Figure 2B). L'analyse de l'orientation du fuseau dans les neuroblastes de lignées invalidées génétiquement pour la huntingtine a montré que la huntingtine est nécessaire au bon déroulement de cette étape. De plus, la surexpression d'un fragment de huntingtine drosophile dans des cellules neuronales murines dépletées en huntingtine rétablit une orientation cor- recte du fuseau. Ainsi, la fonction de la huntingtine dans le positionnement du fuseau de division est conservée chez la drosophile et les mammifères [11]. La découverte de cette fonction devrait non seulement permettre de mieux comprendre les mécanismes fondamentaux de la division cellulaire et de la neurogenèse, mais également ceux qui contribuent à la progression de la maladie de Huntington. $\diamond$

Huntingtin, mitosis and cell fate

\section{CONFLIT D'INTÉRÊTS}

Les auteurs déclarent n'avoir aucun conflit d'intérêts concernant les données publiées dans cet article.

\section{RÉFÉRENCES}

1. Borrell-Pages M, Canals JM, Cordelieres FP, et al. Cystamine and cysteamine increase brain levels of BDNF in Huntington disease via HSJlb and transglutaminase. J Clin Invest 2006 ; 116 : 1410-24.
2. Caviston JP, Ross JL, Antony SM, et al. Huntingtin facilitates dynein/dynactin-mediated vesicle transport. Proc Natl Acad Sci USA 2007 ; $104: 10045-50$

3. Zuccato C, Ciammola A, Rigamonti D, et al. Loss of huntingtin-mediated BDNF gene transcription in Huntington's disease. Science 2001 ; 293 : 493-8.

4. Gauthier LR, Charrin BC, Borrell-Pages M, et al. Huntingtin controls neurotrophic support and survival of neurons by enhancing BDNF vesicular transport along microtubules. Cell $2004 ; 118$ : 127-38.

5. Godin JD, Colombo K, Molina-Calavita M, et al. Huntingtin is required for mitotic spindle orientation and mammalian neurogenesis. Neuron 2010 ; $67: 392-406$

6. Radulescu AE, Cleveland DW. NuMA after 30 years: the matrix revisited. Trends Cell Biol 2010 ; 20 : 214-22

7. Siller KH, Doe CQ. Spindle orientation during asymmetric cell division. Nat Cell Biol 2009; $11: 365-74$.

8. Knoblich JA. Mechanisms of asymmetric stem cell division. Cell 2008 ; 132 : 583-97.

9. Chartier NT, Hyenne V, Labbé JC. Mécanismes de division cellulaire asymétrique : des organismes modèles au développement tumoral. Med Sci (Paris) $2010 ; 26: 251-7$.

10. Romé P, Prigent C, Giet R. Le fuseau mitotique, le centrosome et le cancer : trouvez l'intrus ! Med Sci (Paris) $2010 ; 26: 377-83$.

11. Liévens JC, Birman S. La chorée de Huntington chez la drosophile et chez la souris: vers de nouvelles pistes thérapeutiques? Med Sci (Paris) 2003 ; $19: 593-9$.

\section{NOUVELLE}

\section{Le microARN-16}

\section{Une clé de l'adaptation des neurones à la fluoxétine}

Anne Baudry, Sophie Mouillet-Richard, Benoît Schneider, Jean-Marie Launay, Odile Kellermann

\author{
A. Baudry, S. Mouillet-Richard, B. Schneider, 0. Kellermann : \\ Inserm UMR-S 747-Université Paris Descartes, \\ 75006 Paris, France. \\ J.M. Launay: AP-HP Service de biochimie, \\ Fondation FondaMental, Inserm U942, \\ Hôpital Lariboisière, \\ 2 , rue Ambroise Paré, 75010 Paris, France. \\ odile.kellermann@parisdescartes.fr
}

\section{Le transporteur de la sérotonine: cible d'antidépresseurs comme le Prozac ${ }^{\circledR}$}

Le système sérotoninergique, qui innerve de multiples régions du cerveau, contrôle les comportements alimentaires, sexuels, le cycle veille-sommeil, l'anxiété, l'humeur, etc. [1]. La sérotonine (5-hydroxytryptamine, 5-HT) joue un rôle majeur dans la dépression, même si d'autres neurotransmetteurs comme la noradrénaline sont aussi impliqués. D'ailleurs, des connexions relient les neurones sérotoninergiques situés dans les noyaux du raphé aux neurones noradrénergiques concentrés dans le locus cœruleus (LC)
(Figure 1). Les transporteurs de la sérotonine (SERT, serotonin transporter), de la noradrénaline ou de la dopamine assurent la recapture de ces neurotransmetteurs au niveau de la synapse. En régulant la concentration extracellulaire de ces monoamines, les transporteurs participent à la neurotransmission et sont la cible de nombreuses drogues et agents thérapeutiques (antidépresseurs, antipsychotiques, hallucinogènes, ecstasy, cocaïne, etc.) [2].

Les états dépressifs sont associés à des déficits en 5-HT. Les antidépresseurs développés depuis les années 1960 visent donc principalement à augmenter la concentration en 5-HT au niveau de la synapse pour améliorer ou rétablir la neurotransmission sérotoninergique chez les patients déprimés [3]. Le chef de file des antidépresseurs est la fluoxétine $\left(\right.$ Prozac $^{\circledR}$ ) qui est classée parmi les inhibiteurs sélectifs de la recapture de la sérotonine (SSRI, selective serotonin reuptake inhibitor) (fluoxétine/Prozac ${ }^{\circledR}$, paroxétine, citalopram, sertraline, etc.). Le SERT est donc la cible des SSRI. Néanmoins, quel que soit l'antidépresseur considéré, l'observation clinique montre qu'il faut un délai d'environ trois semaines pour que l'éventuel effet bénéfique de l'antidépresseur soit objectivé. Ce 